University of New Hampshire

University of New Hampshire Scholars' Repository

Space Science Center

Institute for the Study of Earth, Oceans, and

Space (EOS)

2006

\title{
Prospects for GRB Polarimetry with GRAPE
}

\author{
Mark L. McConnell \\ University of New Hampshire - Main Campus, mark.mcconnell@unh.edu \\ Peter F. Bloser \\ University of New Hampshire, Peter.Bloser@unh.edu \\ Jason S. Legere \\ University of New Hampshire, jslegere@unh.edu \\ John R. Macri \\ University of New Hampshire - Main Campus, John.Macri@unh.edu \\ T Narita \\ College of the Holy Cross
}

See next page for additional authors

Follow this and additional works at: https://scholars.unh.edu/ssc

Part of the Astrophysics and Astronomy Commons

\section{Recommended Citation}

Prospects for GRB Polarimetry with GRAPE McConnell, M. L. and Bloser, P. F. and Legere, J. and Macri, J. R. and Narita, T. and Ryan, J. M., AIP Conference Proceedings, 836, 654-659 (2006), DOI:http://dx.doi.org/ $10.1063 / 1.2207970$

This Conference Proceeding is brought to you for free and open access by the Institute for the Study of Earth, Oceans, and Space (EOS) at University of New Hampshire Scholars' Repository. It has been accepted for inclusion in Space Science Center by an authorized administrator of University of New Hampshire Scholars' Repository. For more information, please contact Scholarly.Communication@unh.edu. 


\section{Authors}

Mark L. McConnell, Peter F. Bloser, Jason S. Legere, John R. Macri, T Narita, and James M. Ryan 


\section{AIP $\mid$ Proceedings}

\section{Prospects for GRB Polarimetry with GRAPE}

M. L. McConnell, P. F. Bloser, J. Legere, J. R. Macri, T. Narita, and J. M. Ryan

Citation: AIP Conference Proceedings 836, 654 (2006); doi: 10.1063/1.2207970

View online: http://dx.doi.org/10.1063/1.2207970

View Table of Contents: http://scitation.aip.org/content/aip/proceeding/aipcp/836?ver=pdfcov

Published by the AIP Publishing

Articles you may be interested in

The broadband afterglow of GRB 030328

AIP Conf. Proc. 836, 357 (2006); 10.1063/1.2207921

Future Prospects for High Energy Polarimetry of GammaRay Bursts

AIP Conf. Proc. 727, 589 (2004); 10.1063/1.1810914

The Polarization Evolution of the Optical Afterglow of GRB 030329

AIP Conf. Proc. 727, 269 (2004); 10.1063/1.1810845

High resolution polarimetry of the inner galaxy

AIP Conf. Proc. 609, 20 (2002); 10.1063/1.1471818

The CIPHER telescope for hard $\mathrm{X}$ and soft $\mathrm{y}$-ray polarimetry

AIP Conf. Proc. 510, 809 (2000); 10.1063/1.1303310 


\title{
Prospects for GRB Polarimetry with GRAPE
}

\author{
M. L. McConnell*, P. F. Bloser*, J. Legere*, J. R. Macri*, T. Narita ${ }^{\dagger}$ and J. \\ M. Ryan* \\ * Space Science Center, University of New Hampshire, Durham, NH 03824 \\ ${ }^{\dagger}$ Department of Physics, College of the Holy Cross, Worcester, MA USA
}

\begin{abstract}
This paper discusses the latest progress in the development of GRAPE (Gamma-Ray Polarimeter Experiment), a hard X-ray Compton Polarimeter. The purpose of GRAPE is to measure the polarization of hard X-rays in the $50-300 \mathrm{keV}$ energy range. We are particularly interested in $\mathrm{X}$-rays that are emitted from solar flares and gamma-ray bursts (GRBs). Accurately measuring the polarization of the emitted radiation from these sources will lead to a better understating of both the emission mechanisms and source geometries. The GRAPE design consists of an array of plastic scintillators surrounding a central high- $Z$ crystal scintillator. We can monitor individual Compton scatters that occur in the plastics and determine whether the photon is photo absorbed by the high$\mathrm{Z}$ crystal or not. A Compton scattered photon that is immediately photo absorbed by the high-Z crystal constitutes a valid event. These valid events provide us with the interaction locations of each incident photon and ultimately produces a modulation pattern for the Compton scattering of the polarized radiation. Comparing with Monte Carlo simulations of a 100\% polarized beam, the level of polarization of the measured beam can then be determined. The complete array is mounted on a flat-panel multi-anode photomultiplier tube (MAPMT) that can measure the deposited energies resulting from the photon interactions. The design of the detector allows for a large field-of-view ( $>\pi$ steradian), at the same time offering the ability to be close-packed with multiple modules in order to reduce deadspace. We present in this paper the latest laboratory results obtained from GRAPE using partially polarized radiation sources along with a brief description of our future plans for the GRAPE design.
\end{abstract}

Keywords: X-ray instrumentation, gamma-ray instrumentation, polarimetric instrumentation, gamma-ray bursts

PACS: $95.55 . \mathrm{Ka}, 95.55 . \mathrm{Qf}, 98.70 . \mathrm{Rz}$

\section{INTRODUCTION}

Polarization measurements have become a powerful tool for astronomers throughout the electromagnetic spectrum. It is believed that by accurately measuring hard X-ray polarization levels from solar flares and gamma ray bursts (GRBs) we will be able to better understand both the emission mechanisms and source geometries producing the observed radiation [1]. With this goal in mind, we have been developing a hard X-ray polarimeter design that we call GRAPE (Gamma-Ray Polarimeter Experiment). The purpose of GRAPE is to measure the polarization of hard x-rays in the $50-300 \mathrm{keV}$ energy range. As described here, the GRAPE design is most suitable for studies of either gamma-ray bursts or solar flares, as part of a long-duration balloon platform or as part of a satellite platform. 


\section{THE GRAPE CONCEPT}

The basic physical process used to measure polarization in the 50-300 keV energy range is Compton scattering, in which photons tend to be scattered at a right angle with respect to the incident electric field vector. In the case of an unpolarized beam of incident photons, there will be no net positive electric field vector and therefore no preferred azimuthal scattering angle $(\eta)$; the distribution of scattered photons with respect to $\eta$ will be uniform. However, in the polarized case, the incident photons will exhibit a net positive electric field vector and the distribution in $\eta$ will be asymmetric. The ultimate goal of a Compton scatter polarimeter is to measure the azimuthal modulation pattern of the scattered photons.

The development and design of the GRAPE detector has evolved through three science models [4-12]. Each one represented a successive improvement, but all three essentially operate under the same underlying principle of operation. A high-Z material, the calorimeter, is surrounded by multiple plastic scintillation detectors that serve as a target for the Compton scattering. The plastic scintillators are made of a low-Z material that maximizes the probability of a Compton interaction. The purpose of the calorimeter is to fully absorb the energy of the scattered photon. Ideally, photons that are incident on the plastic scintillator array will Compton scatter only once, and then be subsequently absorbed by the calorimeter. For such an event we measure the energy of the scattered electron in the plastic and the deposited energy of the scattered photon in the calorimeter. With multiple plastic scintillators surrounding the calorimeter, we can determine the azimuthal scatter angle of each valid event. A histogram of these data represents the azimuthal modulation pattern of the scattered photons, which provides a measurement of the polarization parameters (magnitude of the polarization and polarization angle) of the incident flux.

In order to accurately measure the azimuthal modulation (and hence the polarization parameters), we need to correct for geometric effects specific to the individual detector design. When the azimuthal modulation profile is generated, the distribution not only includes the intrinsic modulation pattern due to the Compton scattering process, but it also includes various geometric effects. One of these effects originates from the specific layout of the detector elements within the polarimeter and the associated quantization of possible scatter angles. Other effects include such things as the nonuniform detection efficiency of the PMT used for detector readout. In order to properly account for these effects, the response of the polarimeter must be properly characterized, for example, by measuring the response of the polarimeter to an unpolarized photon beam.

To determine the polarization level, $\mathrm{P}$, of the incident radiation we need to know the modulation factor for a completely polarized beam $\left(\mu_{100}\right)$. We have used simulations based on MGEANT (incorporating the GLEPS polarization code) to model the response of the polarimeter to $100 \%$ polarized incident radiation. The simulations included all important components of the lab setup.

One significant advantage of the GRAPE design is that it affords a very large FoV, with significant sensitivity for sources as far as $60^{\circ}$ off axis. This is ideal for GRB studies, where the source comes from random directions on the sky. Previous estimates of the GRB polarization sensitivity of the GRAPE design $[9,10]$ suggest that a significant number of GRBs could provide useful polarization measurements on a Ultra-Long Du- 


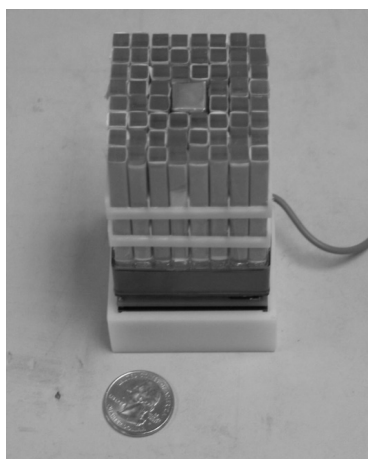

FIGURE 1. GRAPE Science Model 3 (SM3) fully populated with 60 plastic scintillators and a central $\mathrm{CsI}(\mathrm{Na})$ calorimeter.

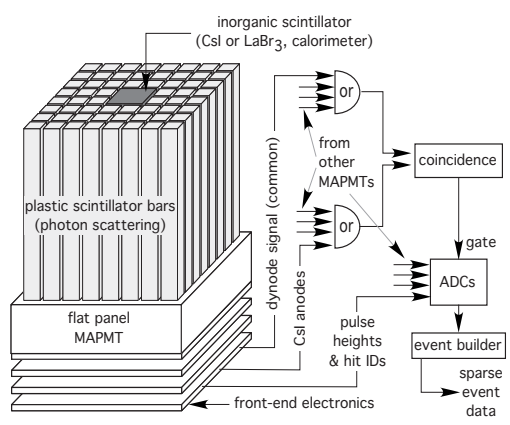

FIGURE 2. GRAPE logic diagram, illustrating the signal processing involved.

ration Balloon (ULDB) platform. Coupled with recent design improvements, we expect that a ULDB version of GRAPE would, depending upon the precise detector configuration, be able to measure GRB polarization at a sensitivity level of about $10 \%$ once every 3-4 days. This sensitivity is far below the polarization level of $80 \%$ reported for GRB021206 [13].

\section{THE GRAPE DESIGN}

The latest version of GRAPE (Science Model 3 - SM3) is a compact design based on the use of a flat-panel MAPMT (Hamamatsu H8500) [11]. The H8500 is a MAPMT with an array of $8 \times 8$ independent anodes. The $5 \mathrm{~mm}$ anodes are arranged with a pitch of $6 \mathrm{~mm}$, occupying a total area of $52 \mathrm{~mm} \times 52 \mathrm{~mm}$. The tube depth is only $28 \mathrm{~mm}$. Each GRAPE module includes a complete array of both plastic scattering elements and a $\mathrm{CsI}(\mathrm{Na})$ calorimeter element mounted on the front surface of the MAPMT (as seen in Figs. 1 and 2). The MAPMT provides readout of both scintillator types. The entire assembly is housed in a light-tight aluminum conatiner. The square design of the MAPMT also allows for the close packing of multiple modules with minimal dead space.

Experiments were conducted in the laboratory in order to determine the effectiveness of the detector. The polarized radiation used in the tests was produced by Compton scattering radiation from a laboratory gamma-ray source [5]. Radiation from a ${ }^{137} \mathrm{Cs}$ source is collimated with lead shielding and directed toward a plastic scintillation detector, referred to as the polarizer. Photons with an initial energy of $662 \mathrm{keV}$ scatter through an angle of $90^{\circ}$ before reaching the polarimeter detector. The radiation reaching the polarimeter is highly polarized (at a level of 55-60\%) and has a reduced energy of 288 $\mathrm{keV}$. The use of the polarizer also allows us to electronically tag photons. A triple coincidence between the polarizer and the two sets of polarimeter detectors provides an efficient means for recording data from the polarized beam.

Initial laboratory tests of SM3 proved unsuccessful. It was discovered that there were light cross-talk issues between the calorimeter and the twelve plastic elements immedi- 

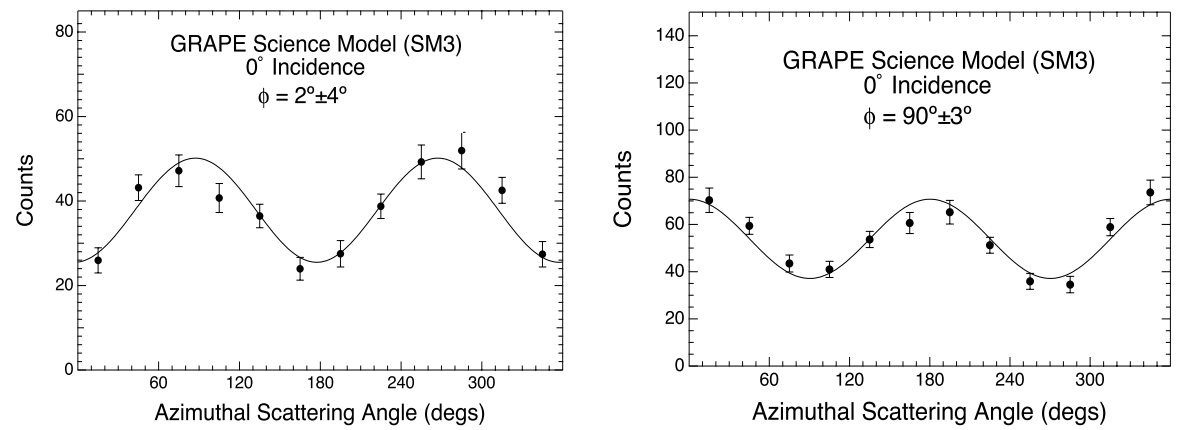

FIGURE 3. Results from SM 3 with the inner plastic ring eliminated from the analysis.

ately surrounding the calorimeter (c.f., Fig. 2). We believe that this can be attributed to the close proximity between the calorimeter and adjacent plastics. With a light output of $\sim 4$ times that of a plastic scintillator, the $\mathrm{CsI}(\mathrm{NaI})$ calorimeter was contributing to the energy recorded in this inner plastic ring. We are currently investigating various options for mitigating this cross talk problem. In the meantime, in order to properly analyze the SM3 data, we decided to eliminate the inner twelve plastic scintillator elements from the analysis. This left 48 plastic elements in the array for inclusion in the data analysis. The data shown in Fig. 3 represent the results of an analysis that excluded the innermost plastic elements. Data was collected for $0^{\circ}$ and $90^{\circ}$ polarization angle and yielded polarization levels of $56( \pm 9) \%$ and $55( \pm 7) \%$ respectively.

\section{GRAPE FUTURE}

The next major step for GRAPE development will be to improve the coincidence timing and energy resolution. We plan to accomplish this by replacing the central CsI calorimeter with one based on Lanthanum Bromide $\left(\mathrm{LaBr}_{3}\right)[14,15]$. This relatively new inorganic scintillator provides an energy resolution that is more than twice as good as $\mathrm{NaI}$ at $662 \mathrm{keV}$ (3\% vs. 7\%). The expected improvement in the GRAPE energy resolution (combining the energy resolution of both the plastic scattering elements and the calorimeter) is shown in Fig. 4. The $\mathrm{LaBr}_{3}$ will also provide better timing characteristics. With decay times of $\sim 25 \mathrm{~ns}$, it is comparable to the plastic scintillator and will greatly improve the coincidence timing characteristics of GRAPE.

In order to provide adequate sensitivity, any realistic application of the GRAPE design would involve an array of polarimeter modules, as in Fig. 5 (possibly surrounded by anti-coincidence shielding). Recent simulations have shown that events involving more than one module in a tiled arrangement will provide a significant increase in detection sensitivity and modulation factor. In addition, we have identified a modified arrangement of plastic and calorimeter elements (splitting the calorimeter into four separate pieces and moving them outward from the center of the array) that will also improve the detection efficiency of a single module by nearly a factor of two. We are now in the process of fabricating a $2 \times 2$ array of GRAPE modules that would be used for 


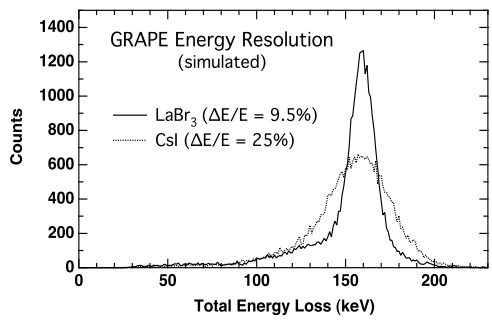

FIGURE 4. Simulated results showing the improved energy resolution that would result from changing the CsI calorimeter to one made from $\mathrm{LaBr}_{3}$.

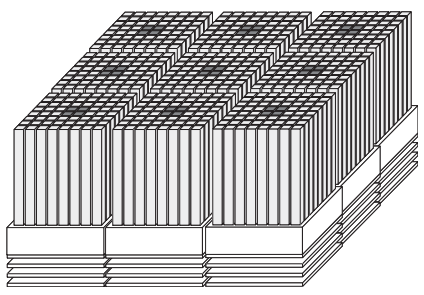

FIGURE 5. An array of nine GRAPE modules.

laboratory testing and that would also serve as a piggyback balloon package to be flown as an engineering test in late 2006.

One possible deployment option for a GRAPE polarimeter array would be as the primary instrument on an Ultra-Long Duration Balloon (ULDB) payload. The ULDB technology can provide balloon flight durations of up to 100 days. A $1 \mathrm{~m}^{2}$ array of GRAPE modules would easily fit within the envelope of a ULDB payload. The ideal configuration for GRB studies would be an array that remains pointed in the vertical direction (i.e., towards the zenith) at all times. In this case, there would be no pointing requirements, only a moderate level of aspect information (continuous knowledge of the azimuthal orientation to $\sim 0.5^{\circ}$ ). An imaging polarimeter could also be designed to match the payload limitations of a ULDB, although the pointing requirements would be much more severe ( $<1^{\circ}$ in both azimuth and zenith). A second deployment option would be as part of a spacecraft payload.

\section{ACKNOWLEDGMENT}

This work is currently supported by NASA grants NNG04GB83G and NNG04WC16G. We would like to thank Mark Widholm and Paul Vachon for their support with the MAPMT design electronics. We would also like to thank the Laboratory for Advanced Instrumentation Research at Embry Riddle, Sparrow Corp., Drew Weisenberger, and Sergio Brambilla for their support with the VME data acquisition setup.

\section{REFERENCES}

1. F. Lei, A.J. Dean and G.L. Hills, Space Sci. Rev. 82, 309-388 (1997).

2. R. Novick, Space Sci. Rev. 18, 389-408 (1975).

3. R. D. Evans, The Atomic Nucleus, McGraw-Hill, New York, 1958.

4. M.L. McConnell, D.J. Forrest, J. Macri, J.M. Ryan, and W.T. Vestrand, "Development of a hard Xray polarimeter for gamma-ray bursts," in AIP Conf. Proc. 428, Gamma-Ray Bursts, edited by C.A. Meegan and P. Cushman, AIP, New York, 1998, pp. 889-893.

5. M.L. McConnell, D.J. Forrest, J. Macri, M. McClish, M. Osgood, J.M. Ryan, W.T. Vestrand and C. Zanes, IEEE Trans. Nucl. Sci. 45, 910-914 (1998). 
6. M.L. McConnell, J.R. Macri, M. McClish, J. Ryan, D.J. Forrest and W.T. Vestrand, IEEE Trans. Nucl. Sci 46, 890 (1999).

7. M.L. McConnell, J.R. Macri, M. McClish, and J. Ryan, SPIE Proc. 3764, 70-78 (1999).

8. M. L. McConnell, J. R. Macri, and J. M. Ryan, "A modular hard X-ray polarimeter for solar flares," inHigh Energy Solar Physics - Anticipating HESSI, edited by R. Ramaty and N. Mandzhavidze, ASP Conf. Ser. 206, ASP, San Francisco, 2000, pp. 280-283.

9. M. L. McConnell, J. R. Ledoux, J. R. Macri, and J. M. Ryan, SPIE Proc. 4851, 1382-1393 (2002).

10. M. L. McConnell, J. R. Ledoux, J. R. Macri, and J. M. Ryan, "The Development of GRAPE, A Gamma-Ray Polarimeter Experiment," in AIP Conf. Proc. 662, Gamma-Ray Burst and Afterglow Astronomy, edited by G. R. Ricker and R. K. Vanderspek, AIP, New York, 2003, pp.503-505.

11. M. L. McConnell, J. R. Ledoux, J. R. Macri, and J. M. Ryan, SPIE Proc. 5165, 334-345 (2004).

12. J. S. Legere, P. Bloser, J. R. Macri, M. L. McConnell, T. Narita and J. M. Ryan, SPIE Proc. 5898, 401-410 (2005).

13. W. Coburn, and S.E. Boggs, Nature 423, 415-417 (2003).

14. van Loef, E. V. D., et al., Appl. Phys. Lett. 79, 1573-1575 (2001).

15. Shah, K. S., et al., IEEE Trans. Nucl. Sci. 50, 2410-2413 (2003). 